\title{
Phaseless inverse scattering in the one-dimensional case R.G. Novikov
}

CNRS (UMR 7641), Centre de Mathématiques Appliquées, Ecole Polytechnique, 91128 Palaiseau, France;

IEPT RAS, 117997 Moscow, Russia

e-mail: novikov@cmap.polytechnique.fr

Abstract. We consider the one-dimensional Schrödinger equation with a potential satisfying the standard assumptions of the inverse scattering theory and supported on the half-line $x \geq 0$. For this equation at fixed positive energy we give explicit formulas for finding the full complex valued reflection coefficient to the left from appropriate phaseless scattering data measured on the left, i.e. for $x<0$. Using these formulas and known inverse scattering results we obtain global uniqueness and reconstruction results for phaseless inverse scattering in dimension $d=1$.

\section{Introduction}

We consider the one-dimensional Schrödinger equation

$$
-\frac{d^{2}}{d x^{2}} \psi+v(x) \psi=E \psi, \quad x \in \mathbb{R}, \quad E>0,
$$

where $v$ satisfies the standard assumptions of the inverse scattering theory (see $[\mathrm{F}]$ ) and is supported on the half-line $x \geq 0$. More precisely, we assume that

$$
\begin{aligned}
& v \text { is real - valued, } v \in L_{1}^{1}(\mathbb{R}), \\
& v(x) \equiv 0 \text { for } x<0,
\end{aligned}
$$

where

$$
L_{1}^{1}(\mathbb{R})=\left\{u \in L^{1}(\mathbb{R}): \int_{\mathbb{R}}(1+|x|)|u(x)| d x<\infty\right\} .
$$

For equation (1.1) we consider the scattering solution $\psi^{+}=\psi^{+}(\cdot, k), k=\sqrt{E}>0$, continuous and bounded on $\mathbb{R}$ and specified by the following asymptotics:

$$
\psi^{+}(x, k)=\left\{\begin{array}{cl}
e^{i k x}+s_{21}(k) e^{-i k x} & \text { as } x \rightarrow-\infty \\
s_{22}(k) e^{i k x}+o(1) & \text { as } x \rightarrow+\infty
\end{array}\right.
$$

for some a priori unknown $s_{21}$ and $s_{22}$. In addition, the coefficients $s_{21}$ and $s_{22}$ arising in (1.4) are the reflection coefficient to the left and transmission coefficient to the right, respectively, for equation (1.1).

In order to find $\psi^{+}$and $s_{21}, s_{22}$ from $v$ one can use well-known results of the onedimensional direct scattering theory, see e.g. [F]. And properties of $\psi^{+}, s_{21}, s_{22}$ are known in detail, see $[\mathrm{DT}],[\mathrm{F}],[\mathrm{HN}],[\mathrm{L}]$.

In particular, it is well-known that

$$
\left|s_{21}(k)\right|^{2}+\left|s_{22}(k)\right|^{2}=1, \quad\left|s_{21}(k)\right|^{2}<1, \quad k>0 .
$$


R.G. Novikov

Let

$$
\left.\mathbb{R}_{+}=\right] 0,+\infty\left[, \quad \mathbb{R}_{-}=\right]-\infty, 0[.
$$

We consider the following two types of scattering data measured on the left for equation (1.1): (a) $s_{21}(k)$ and (b) $\psi^{+}(x, k), \quad x \in X_{-} \subseteq \mathbb{R}_{-}$, where $k=\sqrt{E}>0$.

In addition, we consider the following inverse scattering problems:

Problem 1.1a. Reconstruct potential $v$ on $\mathbb{R}$ from its reflection coefficient $s_{21}$ on $\mathbb{R}_{+}$.

Problem 1.1b. Reconstruct potential $v$ on $\mathbb{R}$ from its scattering data $\psi^{+}(x, \cdot)$ on $\mathbb{R}_{+}$at fixed $x \in \mathbb{R}_{-}$.

Problem 1.2a. Reconstruct potential $v$ on $\mathbb{R}$ from its phaseless scattering data $\left|s_{21}\right|^{2}$ on $\mathbb{R}_{+}$.

Problem 1.2b. Reconstruct potential $v$ on $\mathbb{R}$ from its phaseless scattering data $\left|\psi^{+}\right|^{2}$ on $X_{-} \times \mathbb{R}_{+}$for some appropriate $X_{-}$.

Problem 1.2c. Reconstruct potential $v$ on $\mathbb{R}$ from its phaseless scattering data $\left|s_{21}\right|^{2}$ on $\mathbb{R}_{+}$and $\left|\psi^{+}\right|^{2}$ on $X_{-} \times \mathbb{R}_{+}$for some appropriate $X_{-}$.

Note that in quantum mechanical scattering experiments in the framework of model described by equation (1.1) the phaseless scattering data $\left|s_{21}\right|^{2},\left|\psi^{+}\right|^{2}$ of Problems 1.2a-1.2c can be measured directly, whereas the complex-valued scattering data $s_{21}, \psi^{+}$of Problems 1.1a, 1.1b are not accessible for direct measurements. Therefore, Problems 1.2 are of particular applied interest in the framework of inverse scattering of quantum mechanics. However, Problems 1.1 are much more considered in the literature than Problems 1.2. See $[\mathrm{ChS}],[\mathrm{DT}],[\mathrm{F}],[\mathrm{L}],[\mathrm{M}],[\mathrm{N} 1],[\mathrm{NM}]$ and references therein in connection with Problem 1.1a and $[\mathrm{AS}],[\mathrm{KS}]$ in connection with Problem 1.2a and its modification.

In particular, work $[\mathrm{NM}]$ gives global uniqueness and reconstruction results for Problem 1.1a; see also [AW], [GS] and references given in [AW]. And, obviously, Problem 1.1b is reduced to Problem 1.1a by the formula

$$
s_{21}(k)=e^{i k x} \psi^{+}(x, k)-e^{2 i k x}, \quad x \in \mathbb{R}_{-}, \quad k \in \mathbb{R}_{+} .
$$

On the other hand, for Problem 1.2a it is well known that the phaseless scattering data $\left|s_{21}\right|^{2}$ on $\mathbb{R}_{+}$do not determine $v$ uniquely, in general. In particular, we have that

$$
\begin{aligned}
& s_{21, y}(k)=e^{2 i k y} s_{21}(k), \\
& \left|s_{21, y}(k)\right|^{2}=\left|s_{21}(k)\right|^{2}, \quad k \in \mathbb{R}_{+}, \quad y \in \mathbb{R},
\end{aligned}
$$

where $s_{21}$ is the reflection coefficient to the left for $v$ and $s_{21, y}$ is the reflection coefficient to the left for $v_{y}$, where

$$
v_{y}(x)=v(x-y), \quad x \in \mathbb{R}, \quad y \in \mathbb{R} .
$$

In the present work we continue studies of [N3]. We recall that article [N3] gives, in particular, explicit asymptotic formulas for finding the complex-valued scattering amplitude from appropriate phaseless scattering data for the Schrödinger equation at fixed energy in dimension $d \geq 2$. Then using these formulas related phaseless inverse scattering problems are reduced in [N3] in dimension $d \geq 2$ to well developed inverse scattering 
Formulas for phase recovering from phaseless scattering data at fixed frequency

from the complex-valued scattering amplitude. And, as a corollary, [N3] contains different results on inverse scattering without phase information in dimension $d \geq 2$.

In connection with recent results on phaseless inverse scattering in dimension $d \geq 2$, see also $[\mathrm{K}],[\mathrm{KR} 1],[\mathrm{KR} 2]$, [N2] and references therein.

In order to present results of the present work we use the notations:

$$
\begin{gathered}
S_{1}\left(x_{1}, x_{2}, k\right)=\left\{\left|s_{21}(k)\right|^{2},\left|\psi^{+}(\cdot, k)\right|^{2} \text { on } X_{-}\right\}, \\
\text {where } X_{-}=\left\{x_{1}, x_{2} \in \mathbb{R}_{-}: x_{1} \neq x_{2}\right\}, \quad k \in \mathbb{R}_{+} ; \\
S_{2}\left(x_{1}, x_{2}, x_{3}, k\right)=\left|\psi^{+}(\cdot, k)\right|^{2} \text { on } X_{-}, \\
\text {where } X_{-}=\left\{x_{1}, x_{2}, x_{3} \in \mathbb{R}_{-}: x_{i} \neq x_{j} \text { if } i \neq j\right\}, \quad k \in \mathbb{R}_{+} ; \\
S_{3}(x, k)=\left\{\left|\psi^{+}(x, k)\right|^{2}, \frac{d\left|\psi^{+}(x, k)\right|^{2}}{d x}\right\}, x \in \mathbb{R}_{-}, k \in \mathbb{R}_{+} .
\end{gathered}
$$

Using these notations the main results of the present work can be summarized as follows:

(A1) We give explicit formulas for finding $s_{21}(k)$ from $S_{1}\left(x_{1}, x_{2}, k\right)$ for fixed $x_{1}, x_{2}$ and $k$, where $x_{1} \neq x_{2} \bmod \left(\pi(2 k)^{-1}\right)$; see Theorem 2.1 of Section 2 .

(A2) We give explicit formulas for finding $s_{21}(k)$ from $S_{2}\left(x_{1}, x_{2}, x_{3}, k\right)$ for fixed $x_{1}$, $x_{2}, x_{3}$ and $k$, where $x_{i} \neq x_{j} \bmod \left(\pi k^{-1}\right)$ if $i \neq j$; see Theorem 2.2 of Section 2 .

(A3) We give explicit formulas for finding $s_{21}(k)$ from $S_{3}(x, k)$ for fixed $x$ and $k$; see Theorem 2.3 of Section 2.

(B) We give global uniqueness and reconstruction results (1) for finding $v$ on $\mathbb{R}$ from $S_{1}\left(x_{1}, x_{2}, \cdot\right)$ on $\mathbb{R}_{+}$for fixed $x_{1}, x_{2},(2)$ for finding $v$ on $\mathbb{R}$ from $S_{2}\left(x_{1}, x_{2}, x_{3}, \cdot\right)$ on $\mathbb{R}_{+}$for fixed $x_{1}, x_{2}, x_{3}$, and $(3)$ for finding $v$ on $\mathbb{R}$ from $S_{3}(x, \cdot)$ on $\mathbb{R}_{+}$at fixed $x$; see Theorem 2.4 of Section 2 .

Note that results of (B1)-(B3) follow from (A1)-(A3) and the aforementioned results of $[\mathrm{NM}]$ on Problem 1.1a. In addition, the results of (B1) are global results on Problem $1.2 \mathrm{c}$ and the results of (B2), (B3) are global results on Problem 1.2b.

The main results of the present work are presented in detail in Section 2.

\section{Main results}

We represent $s_{21}$ of (1.4) as follows:

$$
s_{21}(k)=\left|s_{21}(k)\right| e^{i \alpha(k)}, \quad k \in \mathbb{R}_{+} .
$$

We consider

$$
a(x, k)=\left|\psi^{+}(x, k)\right|^{2}-1, \quad x \in \mathbb{R}_{-}, \quad k \in \mathbb{R}_{+},
$$

where $\psi^{+}$is the scattering solutions of (1.4).

Theorem 2.1. Let potential $v$ satisfy (1.2) and $s_{21}$, a be the functions of (1.4), (2.2). Let $x_{1}, x_{2} \in \mathbb{R}_{-}, k \in \mathbb{R}_{+}, x_{1} \neq x_{2} \bmod \left(\pi(2 k)^{-1}\right)$. Then:

$$
\begin{aligned}
& \left|s_{21}\right|\left(\begin{array}{c}
\cos \alpha \\
\sin \alpha
\end{array}\right)=\left(2 \sin \left(2 k\left(x_{2}-x_{1}\right)\right)\right)^{-1} \times \\
& \left(\begin{array}{cc}
\sin \left(2 k x_{2}\right) & -\sin \left(2 k x_{1}\right) \\
-\cos \left(2 k x_{2}\right) & \cos \left(2 k x_{1}\right)
\end{array}\right)\left(\begin{array}{l}
a\left(x_{1}, k\right)-\left|s_{21}\right|^{2} \\
a\left(x_{2}, k\right)-\left|s_{21}\right|^{2}
\end{array}\right),
\end{aligned}
$$


R.G. Novikov

where $\left|s_{21}\right|=\left|s_{21}(k)\right|, \alpha=\alpha(k)$.

Theorem 2.1 is proved in Section 3.

One can see that Theorem 2.1 gives explicit formulas for finding $s_{21}(k)$ from $S_{1}\left(x_{1}, x_{2}, k\right)$ of (1.10) for fixed $x_{1}, x_{2}$ and $k$, where $x_{1} \neq x_{2} \bmod \left(\pi(2 k)^{-1}\right)$.

Theorem 2.2. Let potential $v$ satisfy (1.2) and $s_{21}$, a be the functions of (1.4), (2.2). Let $x_{1}, x_{2}, x_{3} \in \mathbb{R}_{-}, k \in \mathbb{R}_{+}, x_{1} \neq x_{2} \bmod \left(\pi k^{-1}\right)$ if $i \neq j$. Then:

$$
\begin{aligned}
& \left|s_{21}\right|\left(\begin{array}{c}
\cos \alpha \\
\sin \alpha
\end{array}\right)=\left(8\left(\sin \left(k\left(x_{2}-x_{3}\right)\right) \sin \left(k\left(x_{2}-x_{1}\right)\right) \sin \left(k\left(x_{1}-x_{3}\right)\right)\right)^{-1} \times\right. \\
& \left(\begin{array}{cc}
\sin \left(2 k x_{3}\right)-\sin \left(2 k x_{1}\right) & -\sin \left(2 k x_{2}\right)+\sin \left(2 k x_{1}\right) \\
-\cos \left(2 k x_{3}\right)+\cos \left(2 k x_{1}\right) & \cos \left(2 k x_{2}\right)-\cos \left(2 k x_{1}\right)
\end{array}\right)\left(\begin{array}{c}
a\left(x_{2}, k\right)-a\left(x_{1}, k\right) \\
a\left(x_{3}, k\right)-a\left(x_{1}, k\right)
\end{array}\right),
\end{aligned}
$$

where $\left|s_{21}\right|=\left|s_{21}(k)\right|, \alpha=\alpha(k)$.

Theorem 2.2 is proved in Section 3.

One can see that Theorem 2.2 gives explicit formulas for finding $s_{21}(k)$ from $S_{2}\left(x_{1}, x_{2}, x_{3}, k\right)$ of (1.11) for fixed $x_{1}, x_{2}, x_{3}$ and $k$, where $x_{1} \neq x_{2} \bmod \left(\pi k^{-1}\right)$ if $i \neq j$.

Theorem 2.3. Let potential $v$ satisfy (1.2) and $s_{21}, \psi^{+}$be the functions of (1.4). Then the following formulas hold:

$$
\begin{aligned}
& \operatorname{Re}\left(s_{21}(k) e^{-i k x}\right)=-1+\left(\left|\psi^{+}(x, k)\right|^{2}-\left|\operatorname{Im}\left(s_{21}(k) e^{-i k x}\right)\right|^{2}\right)^{1 / 2}, \\
& \operatorname{Im}\left(s_{21}(k) e^{-i k x}\right)=\frac{1}{4 k} \frac{d\left|\psi^{+}(x, k)\right|^{2}}{d x},
\end{aligned}
$$

where $x \in \mathbb{R}_{-}, k \in \mathbb{R}_{+}$, and $(\cdot)^{1 / 2}>0$ in $(2.5)$.

Theorem 2.3 is proved in Section 3.

One can see that Theorem 2.3 gives explicit formulas for finding $s_{21}(k)$ from $S_{3}(x, k)$ of $(1.12)$.

As corollaries of Theorems 2.1, 2.2, 2.3 and results of [NM], we obtain the following global uniqueness and reconstruction results on phaseless inverse scattering for equation (1.1):

Theorem 2.4. Let potential $v$ satisfy (1.2) and $S_{1}, S_{2}, S_{3}$ be the phaseless scattering data of (1.10), (1.11), (1.12). Then: (1) $S_{1}\left(x_{1}, x_{2}, \cdot\right)$ on $\mathbb{R}_{+}$, for fixed $x_{1}, x_{2}$, uniquely determine $v$ on $\mathbb{R}$ via formulas (2.1)-(2.3) and results of $[N M] ;(2) S_{2}\left(x_{1}, x_{2}, x_{3}, \cdot\right)$ on $\mathbb{R}_{+}$, for fixed $x_{1}, x_{2}, x_{3}$, uniquely determine $v$ on $\mathbb{R}$ via formulas (2.1), (2.2), (2.4) and results of $[N M] ;(3) S_{3}(x, \cdot)$ on $\mathbb{R}_{+}$, for fixed $x$, uniquely determine $v$ on $\mathbb{R}$ via formulas $(2.5),(2.6)$ and results of $[N M]$.

\section{Proofs of Theorems $2.1,2.2$ and 2.3}

Proof of Theorem 2.1. Using (1.4) we obtain that

$$
\begin{aligned}
& \left|\psi^{+}(x, k)\right|^{2}=\psi^{+}(x, k) \overline{\psi^{+}(x, k)}= \\
& 1+2 \operatorname{Re}\left(s_{21}(k) e^{-2 i k x}\right)+\left|s_{21}(k)\right|^{2}, \quad x \in \mathbb{R}_{-}, \quad k \in \mathbb{R}_{+} .
\end{aligned}
$$


Formulas for phase recovering from phaseless scattering data at fixed frequency

In addition, in view of $(2.1)$ we have that

$$
2 R e\left(s_{21}(k) e^{-2 i k x}\right)=2\left|s_{21}(k)\right| \cos (2 k x-\alpha(k))
$$

Using (2.2), (3.1), (3.2) we obtain that

$$
\begin{aligned}
& \left|s_{21}(k)\right| \cos (2 k x) \cos (\alpha(k))+\sin (2 k x) \sin (\alpha(k))= \\
& 2^{-1}\left(a(x, k)-\left|s_{21}(k)\right|^{2}\right), \quad x \in \mathbb{R}_{-}, \quad k \in \mathbb{R}_{+} .
\end{aligned}
$$

Using (3.3) for $x=x_{1}$ and $x=x_{2}$, we obtain the system

$$
\begin{aligned}
& \left(\begin{array}{rr}
\cos \left(2 k x_{1}\right) & \sin \left(2 k x_{1}\right) \\
\cos \left(2 k x_{2}\right) & \sin \left(2 k x_{2}\right)
\end{array}\right)\left|s_{21}\right|\left(\begin{array}{c}
\cos \alpha \\
\sin \alpha
\end{array}\right)= \\
& 2^{-1}\left(\begin{array}{l}
a\left(x_{1}, k\right)-\left|s_{21}\right|^{2} \\
a\left(x_{2}, k\right)-\left|s_{21}\right|^{2}
\end{array}\right),
\end{aligned}
$$

where $\left|s_{21}\right|=\left|s_{21}(k)\right|, \alpha=\alpha(k)$.

Formula (2.3) follows from (3.4).

Theorem 2.1 is proved.

Proof of Theorem 2.2. Subtracting equality (3.3) for $x=x_{1}$ from equality (3.3) for $x=x_{2}$ and from equality (3.3) for $x=x_{3}$, we obtain the system

$$
\begin{aligned}
& \left(\begin{array}{cc}
\cos \left(2 k x_{2}\right)-\cos \left(2 k x_{1}\right) & \sin \left(2 k x_{2}\right)-\sin \left(2 k x_{1}\right) \\
\cos \left(2 k x_{3}\right)-\cos \left(2 k x_{1}\right) & \sin \left(2 k x_{3}\right)-\sin \left(2 k x_{1}\right)
\end{array}\right)\left|s_{21}\right|\left(\begin{array}{c}
\cos \alpha \\
\sin \alpha
\end{array}\right)= \\
& 2^{-1}\left(\begin{array}{c}
a\left(x_{2}, k\right)-a\left(x_{1}, k\right) \\
a\left(x_{3}, k\right)-a\left(x_{1}, k\right)
\end{array}\right),
\end{aligned}
$$

where $\left|s_{21}\right|=\left|s_{21}(k)\right|, \alpha=\alpha(k)$.

One can see that

$$
\Delta=\sin \left(2 k\left(x_{3}-x_{2}\right)\right)+\sin \left(2 k\left(x_{2}-x_{1}\right)\right)+\sin \left(2 k\left(x_{1}-x_{3}\right)\right),
$$

where $\Delta$ is the determinant of the system (3.5). In addition, using the formulas

$$
\begin{aligned}
& \sin \varphi_{1}+\sin \varphi_{2}=2 \cos \left(\frac{\varphi_{1}-\varphi_{2}}{2}\right) \sin \left(\frac{\varphi_{1}+\varphi_{2}}{2}\right) \\
& \sin \left(\varphi_{1}+\varphi_{2}\right)=2 \cos \left(\frac{\varphi_{1}+\varphi_{2}}{2}\right) \sin \left(\frac{\varphi_{1}+\varphi_{2}}{2}\right) \\
& \sin \varphi_{1}+\sin \varphi_{2}-\sin \left(\varphi_{1}+\varphi_{2}\right)=4 \sin \left(\frac{\varphi_{1}+\varphi_{2}}{2}\right) \sin \left(\frac{\varphi_{1}}{2}\right) \sin \left(\frac{\varphi_{2}}{2}\right)
\end{aligned}
$$

for $\varphi_{1}=2 k\left(x_{2}-x_{1}\right), \varphi_{2}=2 k\left(x_{1}-x_{3}\right)$, we obtain

$$
\Delta=4 \sin \left(k\left(x_{2}-x_{3}\right)\right) \sin \left(k\left(x_{2}-x_{1}\right)\right) \sin \left(k\left(x_{1}-x_{3}\right)\right) .
$$


R.G. Novikov

Formula (2.4) follows from (3.5), (3.8).

Theorem 2.2 is proved.

Proof of Theorem 2.3. Using (3.1) we obtain that

$$
\left(\operatorname{Re}\left(s_{21}(k) e^{-2 i k x}\right)+1\right)^{2}+\left(\operatorname{Im}\left(s_{21}(k) e^{-2 i k x}\right)\right)^{2}=\left|\psi^{+}(x, k)\right|^{2} .
$$

Formula (2.5), where $(\cdot)^{1 / 2}>0$, follows from (3.9) and the property that $\left|s_{21}(k)\right|<1$, see (1.5).

Using (2.1), (3.1), (3.2) we obtain that

$$
\frac{d\left|\psi^{+}(x, k)\right|^{2}}{d x}=4 k\left|s_{21}(k)\right| \sin (\alpha(k)-2 k x)=4 k \operatorname{Im}\left(s_{21}(k) e^{-2 i k x}\right) .
$$

Formula (2.6) follows from (3.10).

Theorem 2.3 is proved.

\section{References}

[ AS] T. Aktosun, P.E. Sacks, Inverse problem on the line without phase information, Inverse Problems 14, 1998, 211-224.

[AW] T. Aktosun, R. Weder, Inverse scattering with partial information on the potential, J. Math. Anal. Appl. 270, 2002, 247-266.

[ChS] K. Chadan, P.C. Sabatier, Inverse Problems in Quantum Scattering Theory, 2nd edn. Springer, Berlin, 1989

[ DT] P. Deift, E. Trubowitz, Inverse scattering on the line, Comm. Pure Appl. Math. 32, 1979, 121-251.

[ F] L.D. Faddeev, Inverse problem of quantum scattering theory. II, Itogy Nauki i Tekh. Ser. Sovrem. Probl. Mat. 3, 1974, 93-180 (in Russian). English translation in J. Sov. Math. 5, 1976, 334-396.

[GS] F. Gesztesy, B. Simon, Inverse spectral analysis with partial information on the potential. I. The case of an a.c. component in the spectrum, Helv. Phys. Acta 70, 1997, 66-71.

[ HN] G.M. Henkin, R.G. Novikov, Oscillating weakly localized solutions of the Kortewegde Vries equation, Teoret. Mat. Fiz. 61(2), 1984, 199-213 (in Russian); English translation: Theoret. and Math. Phys. 61(2), 1984, 1089-1099.

[K] M.V. Klibanov, Phaseless inverse scattering problems in three dimensions, SIAM J. Appl. Math. 74, 2014, 392-410.

[KR1] M.V. Klibanov, V.G. Romanov, Reconstruction formula for a 3-d phaseless inverse scattering problem for the Schrödinger equation, arXiv:1412.8210v1, December 28, 2014.

[KR2] M.V. Klibanov, V.G. Romanov, Explicit formula for the solution of the phaseless inverse scattering problem of imaging of nano structures, J. Inverse Ill-Posed Probl., DOI 10.1515/jiip-2015-0004

[ KS] M.V. Klibanov, P.E. Sacks, Phaseless inverse scattering and the phase problem in optics, J.Math. Physics 33, 1992, 3813-3821. 
Formulas for phase recovering from phaseless scattering data at fixed frequency

[ L] B.M. Levitan, Inverse Sturm-Liuville Problems, VSP, Zeist, 1987.

[ M] V.A. Marchenko, Sturm-Liuville Operators and Applications, Birkhäuser, Basel, 1986.

[ N1] R.G. Novikov, Inverse scattering up to smooth functions for the Schrödinger equation in dimension 1, Bull. Sci. Math. 120, 1996, 473-491.

[ N2] R.G. Novikov, Explicit formulas and global uniqueness for phaseless inverse scattering in multidimensions, J. Geom. Anal. DOI:10.1007/s12220-014-9553-7; arXiv:1412.5006v1, December 16, 2014.

[ N3] R.G. Novikov, Formulas for phase recovering from phaseless scattering data at fixed frequency, Bull.Sci.Math. (to appear); arXiv:1502.02282v2, February 14, 2015.

[ NM] N.N. Novikova, V.M. Markushevich, On the uniqueness of the solution of the inverse scattering problem on the real axis for the potentials placed on the positive half-axis, Comput. Seismology 18, 1985, 176-184 (in Russian). 